\title{
Barreras para la participación en programas de rehabilitación cardiaca en pacientes sometidos a revascularización percutánea por enfermedad coronaria
}

\author{
Juan Carlos Sánchez-Delgado a,b,*, Adriana Angarita-Fonseca ${ }^{a}$, \\ Adriana Jácome Hortúa ${ }^{a}$, Yésica Malaver-Vega ${ }^{a}$, Erick Schmalbach-Aponte ${ }^{a}$ \\ y Catalina Díaz-Díaz ${ }^{a}$
}

a Universidad de Santander, Bucaramanga, Colombia
b Universidad Santo Tomás Floridablanca, Bucaramanga, Colombia

Recibido el 18 de junio de 2015; aceptado el 28 de agosto de 2015

Disponible en Internet el 11 de noviembre de 2015

\section{PALABRAS CLAVE \\ Enfermedad \\ coronaria; \\ Rehabilitación; \\ Angioplastia; \\ Prevención}

\begin{abstract}
Resumen
Introducción: Los programas de rehabilitación cardiaca promueven cambios de estilo de vida y disminuyen la recurrencia de enfermedad y mortalidad en un $25 \%$. No obstante, la tasa de asistencia a éstos varía entre el 7,5 al $29 \%$, y cerca del 40 al $50 \%$ de los usuarios abandonan el tratamiento en forma prematura.

Objetivo: describir las barreras encontradas en quienes participaron o no en programas de rehabilitación cardíaca después de ser sometidos a una revascularización percutánea.

Métodos: estudio de corte transversal, realizado en treinta sujetos, a quienes se les aplicó la escala de barreras para la rehabilitación cardiaca, que usa preguntas tipo Likert, donde los puntajes más altos indican mayor percepción de barrera para participar en un programa de rehabilitación cardíaca.

Resultados: Las mayores barreras por ítem fueron: «yo encuentro al ejercicio fatigante y/o doloroso», con un promedio de 2,86 , seguido por «Yo hago actualmente ejercicio» y «Yo no sabía de la rehabilitación cardiaca» con un promedio de 2,73. El de menor puntaje fue: «Muchas personas sufren del corazón y no van», con 1,73. Presentaron más barreras quienes no asistieron a un programa de rehabilitación cardíaca $(p<0,05)$ que quienes sí lo hicieron; además las mayores barreras se encontraron en los dominios de necesidades percibidas y comorbilidades/estado funcional $(p<0,05)$. Lo anterior hace suponer falta de orientación y educación respecto a los beneficios que pueden obtener los usuarios al ingresar y adherirse a un programa de
\end{abstract}

\footnotetext{
* Autor para correspondencia.

Correo electrónico: juancarlossanchezd@gmail.com (J.C. Sánchez-Delgado).
} 
rehabilitación cardíaca, independiente de como se sientan o el tipo de limitación funcional que tengan.

(C) 2015 Sociedad Colombiana de Cardiología y Cirugía Cardiovascular. Publicado por Elsevier España, S.L.U. Este es un artículo Open Access bajo la licencia CC BY-NC-ND (http:// creativecommons.org/licenses/by-nc-nd/4.0/).

\section{KEYWORDS}

Coronary disease;

Rehabilitation;

Angioplasty;

Prevention

\section{Barriers for the participation in cardiac rehabilitation programmes in patients undergoing percutaneous revascularisation due to a coronary disease}

\begin{abstract}
Introduction: Cardiac rehabilitation programmes encourage lifestyle changes and reduce the recurrence of morbidity and mortality by $25 \%$. Nevertheless, attendance rate to such programmes varies between 7.5 and $29 \%$, and around 40 to $50 \%$ of users abandon the treatment during the early stages.

Motivation: To describe the barriers found in those who participated or not in cardiac rehabilitation programmes after percutaneous revascularisation.

Methods: Cross-sectional study of 30 patients, who were applied the barrier scale for cardiac rehabilitation, using Likert-type questions, where the highest scores indicated a bigger perception of the barrier to participate in a cardiad rehabilitation programme.

Results: The major barriers per item were: «I find this exercise is tiring and/or painful» with an average of 2.86 , followed by «l currently exercise» and «l did not know about cardiac rehabilitation» with an average of 2.73. The lowest score was: «Many people have heart problems and do not go», with 1.73. More barriers were shown in those who did not attend a cardiac rehabilitation programme $(\mathrm{p}<0.05)$ than whose who did; in addition, most barriers were found in the fields of perceived needs and comorbidities/functional state $(p<0.05)$. This indicates a lack of orientation and education when it comes to the benefits that users could obtain by joining and participating in a cardiac rehabilitation programme, no matter how they feels or the type of functional limitations they may have.

(c) 2015 Sociedad Colombiana de Cardiología y Cirugía Cardiovascular. Published by Elsevier España, S.L.U. This is an open access article under the CC BY-NC-ND license (http:// creativecommons.org/licenses/by-nc-nd/4.0/).
\end{abstract}

\section{Introducción}

La Organización Mundial de la Salud (OMS) señala que las enfermedades cardiovasculares afectan ambos sexos y en la actualidad se han convertido en una problemática de salud pública a la que aún no se le ha brindado la importancia que requiere. Las causas de muerte que preceden a estas afecciones están relacionadas con algunas condiciones modificables, ambientales y comportamentales, tales como el sedentarismo, la obesidad o el tabaquismo, y otras inmutables como la genética o la edad ${ }^{1}$.

En Latinoamérica, específicamente en Brasil, el 61,9\% de los habitantes presenta alguno de estos predictores de riesgo: hipertensión arterial, dislipidemia o diabetes mellitus; en Colombia, se estima que las enfermedades cardiovasculares son la tercera causa de fallecimiento en una población socioeconómicamente activa, relacionada con los estilos de vida poco saludables adquiridos durante la infancia ${ }^{2}$.

El impacto de estos malos hábitos repercute en la salud de las personas y perjudica la calidad de vida de quienes no controlan sus factores de riesgo. En el caso de los individuos que son intervenidos mediante cirugía tras un evento coronario, la OMS recomienda el uso de los programas de rehabilitación cardiaca, la cual se define como el conjunto de actividades necesarias para asegurar a los cardiópatas una condición física, mental y social óptima que les permita ocupar por sus propios medios un lugar tan normal como les sea posible en la sociedad ${ }^{1}$. La rehabilitación cardíaca incluye un manejo integral desarrollado por diferentes profesionales de la salud especialistas en el área $^{3}$; adicionalmente The Scottish Intercollegiate Network menciona que los programas de rehabilitación cardíaca incluyen cuatro fases cuyo objetivo es aumentar gradualmente la capacidad funcional y mantenerla, a fin de mejorar la calidad de vida de estas personas ${ }^{4}$.

Así pues, los programas de rehabilitación cardiaca comprenden una serie de actividades que incluyen ejercicio físico, cuidado nutricional, seguimiento psicológico y cuidado de los factores de riesgo, lo cual puede disminuir en un $25 \%$ las tasas de morbilidad y mortalidad cardiovascular ${ }^{2}$. No obstante, a pesar de los beneficios que se obtienen gracias a estos programas, Corotto et al. reportan que menos del $30 \%$ de la población apta ingresa a este servicio y aproximadamente el $50 \%$ deserta de manera prematura. La adherencia a los programas de rehabilitación cardíaca, según la American Heart Association (AHA), se define como el porcentaje de dosis prescritas tomadas durante un periodo específico de tiempo. Oćonnor et al. determinaron que el número de sesiones durante la fase ॥ de un programa de rehabilitación cardíaca es de 36, y que la adherencia en esta fase está condicionada por una asistencia igual o mayor de 24 sesiones. 
Se considera que los resultados más exitosos se obtienen cuando los pacientes acuden por lo menos con un porcentaje igual o superior del $80 \%$ de las sesiones programadas ${ }^{5,6}$.

De otra parte, Mclarck et al., identificaron la existencia de barreras contextuales y personales que impiden la participación en los programas de rehabilitación cardíaca. Dichas limitaciones hacen referencia al conjunto de situaciones del paciente como creencias acerca del manejo de su enfermedad, el medio en el que vive, la distancia que debe recorrer desde donde se encuentre hasta el sitio del programa, las situaciones laborales y la atención del sistema de salud al que pertenece ${ }^{7}$.

Ante la escasa evidencia e inexistencia de un instrumento local que describa las barreras personales y contextuales del paciente cardiópata para no asistir o no terminar su proceso de rehabilitación, se seleccionó un instrumento denominado Escala de Barreras para Rehabilitación Cardiaca (EBRC) diseñado en Toronto-Ontario Canadá, el cual tiene una confiabilidad aceptable en sus versiones inglesa (alpha de cronbach de $0,88, \mathrm{CCl}=0,64$ ) y portuguesa (alpha de cronbach de $0,88, \mathrm{CCl}=0,68)^{8-10}$, además nuestro grupo de investigación realizó la adaptación transcultural de la escala y determinó su validez a raíz de un índice de validez de contenido para la relevancia y para la pertinencia de 0,86 y 0,88 , respectivamente ${ }^{11}$. El propósito de este trabajo es aplicar la EBRC y describir las barreras encontradas en quienes participaron o no en programas de rehabilitación cardíaca después de revascularización percutánea en Los Comuneros Hospital Universitario de Bucaramanga.

\section{Materiales y métodos}

Estudio de corte transversal realizado en sujetos mayores de 18 años, a quienes, a causa de un evento isquémico coronario, se les hizo revascularización coronaria, entre enero y julio de 2014, en Los Comuneros Hospital Universitario de Bucaramanga-Colombia. Se excluyeron analfabetas o aquellos que presentaran algún tipo de limitación mental que les impidiera diligenciar la EBRC.

Con base en los criterios anteriores se realizó una búsqueda en la base de datos del servicio de hemodinámica del hospital en mención conformando una población elegible de 98 usuarios, quienes fueron contactados vía telefónica, mediante tres llamadas en horas de la mañana y la tarde; al tercer intento se dio por perdida la persona. De 98, se excluyeron 33, que no se pudieron contactar; 24 vivían en lugares de difícil ubicación, 5 se negaron a participar y 6 habían fallecido. Se conformó así una muestra de 30 pacientes que fueron visitados en sus domicilios para autodiligenciar el instrumento EBRC.

Dentro de las variables evaluadas figuran las características generales de la población (sexo, edad, lugar de procedencia, estado civil, estrato socio-económico, régimen de salud, situación laboral, nivel de escolaridad y tipo de discapacidad), las relacionadas con el programa de rehabilitación cardíaca (participación o no en un programa de rehabilitación cardíaca, número de sesiones) y las variables clínicas.

Las barreras se evaluaron con la EBRC, la cual es de tipo Likert de 5 puntos: 1 = En muy desacuerdo, 2 = En desacuerdo, 3 =Ni desacuerdo, $\mathrm{Ni}$ acuerdo, 4=En acuerdo; y
$5=$ muy de acuerdo. Un puntaje elevado indica mayor barrera para la participación en los programas de rehabilitación cardíaca. Específicamente, está compuesta por 21 ítems, que se categorizan en cuatro dominios: comorbilidades/funcionalidad, necesidades percibidas, problemas personales/familiares y viaje/conflicto de trabajo.

\section{Análisis estadístico}

Se realizó doble digitación en el programa Epidata 3.1 para la introducción de los datos recogidos; además se utilizó el programa Stata 13.1 para el análisis de los datos. Se calcularon medidas de tendencia central y de dispersión para las variables cuantitativas, y frecuencias absolutas y relativas para las variables cualitativas. Las diferencias en el puntaje de cada dominio y en el puntaje total según participación en un programa de rehabilitación cardíaca se evaluaron mediante la prueba $\mathrm{t}$ de Student. El nivel de significancia usado fue 0,05 .

\section{Resultados}

En la tabla 1 se muestran las características de la población de estudio. El 66,6\% eran hombres, con promedio de edad de $67,0 \pm 11,0$ años; el 73,3\% de procedencia urbana, el 36,7\% casados/as, el $80 \%$ pertenecía a un régimen de salud contributivo, al $76,66 \%$ le realizaron angioplastia con stent y el 63,33\% participó en un programa de rehabilitación cardiaca, de los cuales un $50 \%$ asistió de 12 a 23 sesiones. El profesional que mayor participación tuvo en el proceso de rehabilitación fue el fisioterapeuta con un $90 \%$.

En la tabla 2 se observan las características clínicas de la población evaluada. Los factores de riesgo más frecuentes fueron hipertensión arterial (80\%), extabaquismo $(60 \%)$ y dislipidemia $(53,33 \%)$, además, la arteria más comprometida fue la descendente anterior con $(56,66 \%)$ mientras que el infarto agudo de miocardio con elevación del segmento ST fue el tipo más común.

La tabla 3 muestra que las principales barreras para acceder a los programas de rehabilitación cardíaca fueron encontrar al «Ejercicio fatigante o doloroso» con un promedio 2,86, seguido por «Yo hago actualmente ejercicio en mi casa o en mi comunidad», «Yo no sabía de la rehabilitación cardiaca» con un promedio de $2,73, y$ «costos» con 2,66. Los menores puntajes de barreras fueron: «Yo creo que fui remitido pero no me llamaron» con promedio de 2, «Me siento viejo», «Paso mucho tiempo para ser remitido» con promedio 1,96, «Tengo otros motivos para no ir a rehabilitación cardiaca» con 1,75 y «Muchas personas sufren del corazón y no van», con el menor puntaje.

En la tabla 4 se muestran los resultados agrupados por dominios, para quienes asistieron y quienes no a un programa de rehabilitación cardíaca. Los sujetos que no asistieron presentan más barreras que quienes sí lo hicieron, en los dominios de percepción de salud y comorbilidades, con resultados estadísticamente significativos $(p<0,05)$. Al comparar las medias totales de la escala por grupos, se observan mayores barreras en quienes no participaron en un programa de rehabilitación cardíaca $(p=0,006)$. 
Tabla 1 Caracterización de la población de estudio

\begin{tabular}{|c|c|c|c|}
\hline \multicolumn{2}{|c|}{ Variable } & \multirow{2}{*}{$\begin{array}{l}\mathrm{n} \\
10\end{array}$} & \multirow{2}{*}{$\frac{\%}{33,3}$} \\
\hline Género & Femenino & & \\
\hline & Masculino & 20 & 66,6 \\
\hline \multirow[t]{2}{*}{ Edad } & Media & 67,2 & \\
\hline & SD & 10,94 & \\
\hline \multirow[t]{2}{*}{ Lugar de procedencia } & Rural & 8 & 26,6 \\
\hline & Urbana & 22 & 73,3 \\
\hline \multirow{6}{*}{ Estado civil } & Soltero & 2 & 6,6 \\
\hline & Viudo & 4 & 13,3 \\
\hline & Unión libre & 4 & 13,3 \\
\hline & Casado & 17 & 56,6 \\
\hline & Divorciado & 2 & 6,6 \\
\hline & No responde & 1 & 3,3 \\
\hline \multirow[t]{3}{*}{ Estrato socioeconómico } & Estrato uno y dos & 11 & 36,6 \\
\hline & Estrato tres y cuatro & 17 & 56.6 \\
\hline & Estrato seis & 2 & 6,6 \\
\hline \multirow[t]{4}{*}{ Régimen de salud } & Subsidiado & 4 & $13,33 \%$ \\
\hline & Contributivo & 23 & $76,67 \%$ \\
\hline & Régimen especial & 1 & $3,33 \%$ \\
\hline & Otro & 2 & $6,66 \%$ \\
\hline \multirow[t]{4}{*}{ Situación laboral } & Empleado & 6 & $20 \%$ \\
\hline & Hogar & 9 & $30 \%$ \\
\hline & Jubilado y/o pensionado por discapacidad & 12 & $40 \%$ \\
\hline & Desempleado & 3 & $10 \%$ \\
\hline \multirow[t]{5}{*}{ Nivel de escolaridad } & Ed. básica primaria & 15 & $50 \%$ \\
\hline & Ed. básica secundaria & 6 & $20 \%$ \\
\hline & Técnico & 4 & $13,33 \%$ \\
\hline & Tecnológico & 2 & $6,67 \%$ \\
\hline & Profesional & 3 & $10 \%$ \\
\hline \multirow[t]{2}{*}{ Vive } & Solo & 3 & $10 \%$ \\
\hline & Acompañado & 27 & $90 \%$ \\
\hline \multirow[t]{2}{*}{ Discapacidad física } & No & 27 & $90 \%$ \\
\hline & Sí & 3 & $10 \%$ \\
\hline \multirow[t]{3}{*}{ Tipo de discapacidad } & Gonartrosis bilateral & 1 & $33,33 \%$ \\
\hline & Parkinson & 1 & $33,33 \%$ \\
\hline & Amputación & 1 & $33,33 \%$ \\
\hline \multirow{2}{*}{$\begin{array}{l}\text { Participación en programas de } \\
\text { rehabilitación cardíaca }\end{array}$} & No & 11 & $36,67 \%$ \\
\hline & Sí & 19 & $63,33 \%$ \\
\hline \multirow[t]{5}{*}{ Número de sesiones asistidas } & 1 a 11 sesiones & 6 & 31,5 \\
\hline & 12 a 23 sesiones & 10 & 52,6 \\
\hline & 24 a 35 sesiones & 1 & 5,2 \\
\hline & 36 sesiones & 1 & 5,2 \\
\hline & Otro & 1 & 5,2 \\
\hline \multirow{6}{*}{$\begin{array}{l}\text { Profesionales que participaron en } \\
\text { la rehabilitación cardíaca }\end{array}$} & Fisioterapia & 18 & 94,7 \\
\hline & Psicología & 7 & 36,8 \\
\hline & Nutrición & 14 & 73,6 \\
\hline & Cardiología & 15 & 78,9 \\
\hline & Enfermería & 11 & 57,8 \\
\hline & Otro & 1 & 5,2 \\
\hline
\end{tabular}

\section{Discusión}

Los resultados demuestran que el género masculino es la población más afectada, lo cual concuerda con la literatura revisada ${ }^{12-20}$. Así mismo, Tsai et al. y Arrigo et al. ${ }^{13,14}$ demuestran que la hipertensión arterial es la comorbilidad más frecuente en los pacientes cardiópatas, lo cual está en consonancia con los resultados de nuestra investigación. En cuanto a la participación en los programas de rehabilitación cardíaca, la mayor parte de la población evaluada accedió a este servicio, lo cual no concuerda con los resultados de las investigaciones revisadas, ya que éstas afirman que aproximadamente el $30 \%$ de la población apta para participar en estos programas, lo hace ${ }^{2-22}$. 
Tabla 2 Características clínicas

\begin{tabular}{llll}
\hline & Variable & & $\mathrm{n}$ \\
\hline Tipo de angioplastia & Convencional & 1 & 3,3 \\
& Con stent & 29 & 96,9 \\
Infarto agudo de miocardio & CEST & 22 & 73,3 \\
Vaso comprometido & SEST & 8 & 26,6 \\
& DA & 17 & 56,6 \\
Factores de riesgo & CD & 11 & 36,6 \\
& Circunfleja & 5 & 16,6 \\
& Infarto previo & 13 & 43,3 \\
& HTA & 24 & 30 \\
Fracción de eyección & DM & 9 & 30 \\
& Dislipidemias & 16 & 53,3 \\
& Tabaquismo & 3 & 10 \\
& Extabaquismo & 18 & 26 \\
\end{tabular}

IAM: infarto agudo de miocardio; CEST: con elevación del segmento ST; SEST: sin elevación del segmento ST; DA: descendente anterior; CD: coronaria derecha; HTA: hipertensión arterial; DM: diabetes mellitus.

En lo referente al número de sesiones de rehabilitación cardíaca realizadas, solo el $5,2 \%$ hizo entre 24 y 35 sesiones y el 5,2\%, 36 sesiones, lo cual es coherente con los estudios revisados que refieren que cerca del $50 \%$ de los usuarios no terminan el tratamiento. Ejemplo de ello es el estudio de Flórez et al. ${ }^{5}$, quienes registraron que sólo el $22 \%$ de la muestra terminó de manera satisfactoria la fase ॥ de rehabilitación cardíaca.

En otro punto la mayor barrera percibida fue: «yo encuentro al ejercicio fatigante y/o doloroso», «yo hago actualmente ejercicio en mi casa o comunidad», «yo no sabía de la rehabilitación cardiaca» y «costos», lo cual es coherente con los hallazgos descritos por Grace et al. ${ }^{23}$ quienes identificaron las mayores barreras de la población evaluada en el ítem: «Yo hago ejercicio en mi casa o en mi comunidad»; así mismo, Ghisi et al. ${ }^{24}$ realizaron la valoración de las barreras en dos ciudades (Luzerna, Florianópolis) de Brasil, y evidenciaron que una de las barreras más influyentes fue «Yo no sé sobre la rehabilitación cardíaca». Por otra parte, contrario a lo que se reporta en la literatura,

Tabla 3 Escala de Barreras para Rehabilitación Cardíaca

\begin{tabular}{|c|c|c|c|c|c|}
\hline Afirmaciones de EBRC & Promedio & Desviación estándar & Mediana & $\mathrm{P} 25$ & P75 \\
\hline Distancia & 2,5 & 1,7 & 2 & 1 & 4 \\
\hline Costos & 2,7 & 1,7 & 2 & 1 & 4 \\
\hline Transporte & 2,6 & 1,6 & 2,5 & 1 & 4 \\
\hline Responsabilidades familiares & 2,2 & 1,5 & 1 & 1 & 3 \\
\hline Yo no sabía de la rehabilitación cardiaca & 2,7 & 1,6 & 3 & 1 & 4 \\
\hline Yo no necesito la rehabilitación cardiaca & 2,1 & 1,3 & 1,5 & 1 & 3 \\
\hline Yo hago actualmente ejercicio & 2,7 & 1,6 & 2 & 1 & 4 \\
\hline Condiciones climáticas & 2,1 & 1,5 & 1 & 1 & 4 \\
\hline Yo encuentro al ejercicio fatigante $\mathrm{y} / \mathrm{o}$ doloroso & 2,9 & 1,5 & 2 & 2 & 4 \\
\hline Falta de tiempo & 2,3 & 1,5 & 1,5 & 1 & 4 \\
\hline Responsabilidades & 2,1 & 1,4 & 1 & 1 & 3 \\
\hline Falta de energía emocional y/o física & 2,3 & 1,4 & 2 & 1 & 4 \\
\hline Otros problemas de salud & 2,3 & 1,5 & 2 & 1 & 4 \\
\hline Me siento viejo & 2,0 & 1,2 & 1 & 1 & 3 \\
\hline Mi doctor no me remitió & 2,5 & 1,6 & 2 & 1 & 4 \\
\hline Muchas personas sufren del corazón y no van & 1,7 & 1,0 & 1 & 1 & 2 \\
\hline Yo puedo manejar mi problema y no necesito ayuda & 2,0 & 1,3 & 1,5 & 1 & 3 \\
\hline Yo creo que fui remitido pero no me llamaron & 2 & 1,3 & 1 & 1 & 3 \\
\hline Paso mucho tiempo para ser remitido & 2,0 & 1,4 & 1 & 1 & 3 \\
\hline Yo prefiero cuidar mi salud solo y no en grupo & 2,4 & 1,5 & 2 & 1 & 4 \\
\hline Tengo otros motivos para no ir a rehabilitación cardiaca & 1,8 & 1,3 & 1 & 1 & 2 \\
\hline
\end{tabular}


Tabla 4 Media de los dominios de la escala barreras de la rehabilitación cardiaca según participación en un programa de rehabilitación cardíaca

\begin{tabular}{lllll}
\hline Dominio & $\begin{array}{l}\text { No participo en programas de } \\
\text { rehabilitación cardíaca }(\mathrm{n}=11)\end{array}$ & $\begin{array}{l}\text { Sí participo en programas de } \\
\text { rehabilitación cardíaca }(\mathrm{n}=19)\end{array}$ & Total & Valor $\mathrm{p}$ \\
\hline Percepción de salud & $2,7 \pm 0,9$ & $2,0 \pm 0,8$ & $2,3 \pm 0,9$ & 0,029 \\
Factores logísticos & $2,4(1,8-3,6)$ & $1,4(1,0-3,6)$ & $2,2(1,0-3,6)$ & 0,304 \\
Conflictos de trabajo & $2,5 \pm 1,4$ & $2,1 \pm 1,5$ & $2,2 \pm 1,4$ & 0,464 \\
Comorbilidades & $3,5(2,0-4,0)$ & $1,8(1,3-2,5)$ & $2,0(1,3-3,3)$ & 0,007 \\
Total & $2,8 \pm 0,7$ & $2,0 \pm 0,6$ & $2,3 \pm 0,7$ & 0,005 \\
\hline
\end{tabular}

el ítem «mi doctor no me remitió», parece no ser la mayor barrera de los sujetos evaluados para participar en un programa de rehabilitación cardíaca ${ }^{25}$.

Se obervó que quienes no asistieron a un programa de rehabilitación cardíaca, refirieron mayores barreras que los que sí asistieron, así como también que el estado funcional y las necesidades percibidas, son las barreras más importantes para no acceder a los servicios de rehabilitación cardíaca, lo cual coincide con los resultados del trabajo realizado por Barros et al. ${ }^{26}$ donde el grupo que no asistió a un programa de rehabilitación cardíaca presentó barreras más significativas en estos mismos dominios $(p<0,001)$.

El dominio de estado funcional incluye los ítems «Yo encuentro el ejercicio fatigante $\mathrm{y} / \mathrm{o}$ doloroso», $\mathrm{y}$ «falta de energía», lo cual puede estar relacionado con el miedo del paciente a moverse después de haber presentado la enfermedad. Bäck et al. ${ }^{27}$ encontraron que el $20 \%$ de los sujetos evaluados con enfermedad coronaria, presentaba temor al movimiento, incluso seis meses después del evento cardiaco, lo cual puede afectar de manera negativa el proceso de rehabilitación; de ahí que sea, importante reconocer la kinesiofobia o «temor al movimiento» en los programas de prevención, ya que esta condición puede afectar los procesos de rehabilitación ${ }^{28}$.

\section{Conclusión}

Los pacientes que no participaron en un programa de rehabilitación cardíaca tuvieron mayores barreras en comparación con quienes sí lo hicieron. Las principales barreras para participar fueron las referentes a los dominios de comorbilidades y percepción de salud. Lo anterior supone falta de orientación y educación respecto a los beneficios que pueden obtener los usuarios al ingresar y adherirse a un programa de rehabilitación cardíaca, de manera independiente de cómo se sientan o del tipo de limitación funcional que tengan.

\section{Limitaciones}

De la población elegible, 33 sujetos fueron excluidos por imposibilidad para ser contactados y 24 por dificultades de acceso al lugar de residencia, lo cual puede considerarse como una posible barrera para el acceso al servicio de rehabilitación cardíaca y plantear un sesgo que requiere mención.

\section{Responsabilidades éticas}

Protección de personas y animales. Los autores declaran que los procedimientos seguidos se conformaron a las normas éticas del comité de experimentación humana responsable y de acuerdo con la Asociación Médica Mundial y la Declaración de Helsinki.

Confidencialidad de los datos. Los autores declaran que han seguido los protocolos de su centro de trabajo sobre la publicación de datos de pacientes.

Derecho a la privacidad y consentimiento informado. Los autores han obtenido el consentimiento informado de los pacientes y/o sujetos referidos en el artículo. Este documento obra en poder del autor de correspondencia.

\section{Financiación}

Esta investigación fue realizada con recursos de la Universidad de Santander UDES. Proyecto de convocatoria 017-13.

\section{Conflicto de intereses}

Los autores declaran no tener conflictos de interés.

\section{Agradecimientos}

Los autores agradecen a la Facultad de Salud y al programa de Fisioterapia de la Universidad de Santander UDES, por su apoyo y acompañamiento en la realización de la presente investigación.

\section{Bibliografía}

1. World health organization: technical report series 270 . Rehabilitation of patients with cardiovascular disease. Génova; 1964.

2. Mair V, Breda A, Boquembuzo, Nagem L. Avaliacao da aderencia ao programa de rehabilitacao cardiaca em um hospital particular geral. Einstein (Sao Paulo). 2013;11(3):278-84.

3. Myers J, Prakash M, Froelicher V, Partington S, Atwood J. Exercise capacity and mortality among men referred for exercise testing. N Eng J Med. 2002;346(11):793-801.

4. Martin A, Woods $C$. What sustains long-term adherence to structured physical activity after a cardiac event. J Ageing and Physical Activity. 2012;20:135-47. 
5. Flórez N, Rocha M, Rodríguez A, Toba K. Evidencia en la adherencia al programa de rehabilitación cardiaca de la fundación Cardioinfantil. Bogotá; 2005-2009.

6. Corotto P, McCarey M, Adams S, Khazanie P, Whellan D. Heart failure patient adherence, epidemiology, cause, and treatment. Heart Failure Clin. 2013;9:49-58.

7. Clark A, King K, Spaling M. Factors influencing participation in cardiac rehabilitation programmes after referral and initial attendance: qualitative systematic review and meta-synthesis. Clin Rehab. 2013;27(10):948-59.

8. Grace Sherry York University. CRBS Development (en línea). Disponible en: http://www.yorku.ca/sgrace/crbarriersscale/ pproperties.html [Acceso 21 Nov 2014].

9. Shanmugasegaram S, Gagliese L, Oh P, Stewart DE, Brister SJ, Chan V, et al. Psychometric validation of the cardiac rehabilitation barriers scale. Clin Rehab. 2011;26(2):152-64.

10. Melo L, Dos Santos R, Schveitzer V, Barros A, Recchi TL, Oh P, et al. Desarrollo y validación de la versión en portugués de la escala de barreras para rehabilitación cardíaca. Arq Bras Cardiol. 2012;98(4):344-52.

11. Sánchez J, Angarita A, Jácome A, Pinzón S. Validez de contenido de la escala de barreras para la rehabilitación cardiaca. Revista Universidad y Salud. 2015; 17 (2).

12. Anchique C, Pérez C, López F, Cortes M. Estado actual de la rehabilitación cardiovascular en Colombia (2010). Rev Colomb Cardiol. 2011;18.(6).

13. Tsai Mei W, Chie WC, Kuo TB, Chen MF, Liu JP, Chen TT, et al. Effects of exercise training on heart rate variability after coronary angioplasty. Physical Therapy. 2006;86(5):626-35.

14. Arrigo I, Brunner H, Lefkovits M, Pfisterer M, Hoffman A. Comparative outcome one year after formal cardiac rehabilitation: the effects of a randomized intervention to improve exercise adherence. Eur Soc Cardiol. 2008;15:306-11.

15. Naranjo N, Díaz F, Garcia R. Influencia de la rehabilitación cardíaca sobre la tasa de rehospitalización en pacientes con infarto agudo de miocardio, Santander, Colombia. Rev Salud Pública. 2012;14(5):831-41.

16. Sundararajan V, Bunker S, Begg S, Helen M. Attendance rates and outcomes of cardiac rehabilitation in Victoria, 1998. MJA. 2014;180:268-71.

17. Scane K, Alter D, Oh P, Brooks D. Adherence to a cardiac rehabilitation home program model of care: a comparison to a well- established traditional on-site supervised programme. NRC Research Press. 2012;37:206-13.

18. Luszczynska A, Sutton S. Physical activity after cardiac rehabilitation: evidence that different types of self-efficacy are important in maintainers and relapsers. Rehabilitation Physical. 2006;51(4):314-21.

19. Haddadzadeh MH, Maiya AG, Padmakumar RS, Bijan MF. Effect of exercise based cardiac rehabilitation on ejection fraction in coronary artery disease patients: a randomized controlled trial. Heart Views. 2011;12:51-7.

20. Meillier L, Nielsen K, Larsen F, Larsen M. Socially differentiated cardiac rehabilitation: Can we improve referral, attendance and adherence among patients with first myocardial infarction. Scandinavian J Pub Health. 2012;40: 286-93.

21. Moradi B, Esmaeilzadeh M, Maleki M, Sari L. Factors associated with failure to complete phase II cardiac rehabilitation: survey registry in rajaie cardiovascular medical and research center. Int Cardiovasc Res J. 2011;5(4):139-42.

22. Castillo M, Vivas M, Pinedo R, Jiménez J. Rehabilitación cardiaca en el síndrome coronario agudo. Rehabilitación (Madrid). 2006;40(6):318-32.

23. Grace SL, Gravely-Witte S, Kayaniyil S, Brual J, Suskin N, Stewart DE. A multisite examination of sex differences in cardiac rehabilitation barriers by participation status. J Womens Health (Larchmt). 2009;18(2):209-16.

24. Ghisi GL, Santos RZ, Aranha EE, Nunes AD, Oh P, Benetti M, et al. Perceptions of barriers to cardiac rehabilitation use in Brazil. Vasc Health Risk Manag. 2013;9:485-91.

25. Menezes R. Cardiac rehabilitation and exercise therapy in the elderly: Should we invest in the aged? J Geriatric Cardiol. 2012;9:68-75

26. Barros Aline, Zulianello R, Decker C, Melo G, Grace S, Benetti M. Diferentes barreiras para reabilitação cardiaca. Rev Bras Cardiol. 2014; 27(4). Comunicação Preliminar.

27. Bäck M, Cider A, Herlitz J, Lundberg M, Jansson B. The impact on kinesiophobia (fear of movement) by clinical variables for patients with coronary artery disease. Int J Cardiol. 2013;167:391-7.

28. Kocjan J, Knapik A. Barriers of physical activity (kinesiophobia) in patients subjected to cardiac rehabilitation. Baltic J Health Physical Activity. 2014;6(4):291-7. 